\title{
Intervenção fisioterapêutica em adultos roncadores
}

\author{
Physiotherapeutic intervention in snoring adults
}

\author{
Francine de Aguiar ${ }^{[a]}$, Rosita Espíndola Santos $\operatorname{Rech}^{[b]}$, Kelser de Souza Kock ${ }^{[c]}$
}

[a] Fisioterapeuta, Universidade do Sul de Santa Catarina (Unisul), Tubarão, SC - Brasil, e-mail: fram.aguiar@gmail.com

[b] Fisioterapeuta, Universidade do Sul de Santa Catarina (Unisul), Tubarão, SC - Brasil, e-mail: rositarech@gmail.com

[c] Fisioterapeuta, Especialista em Fisiologia do Exercício, Universidade do Sul de Santa Catarina (Unisul), Tubarão, SC - Brasil, e-mail:kelserkock@yahoo.com.br

\section{Resumo}

Introdução: O ronco é caracterizado como o som emitido pela vibração dos tecidos moles da faringe, por causa da dificuldade que o ar encontra ao passar pela via aérea superior com obstrução parcial. Objetivo: Analisar a eficácia da intervenção fisioterapêutica em adultos roncadores, atendidos na clínicaescola de Fisioterapia da Universidade do Sul de Santa Catarina (Unisul). Método: Trata-se de uma pesquisa explicativa, quantitativa e quase experimental. Na primeira fase, a amostra foi composta por sete indivíduos residentes da cidade de Tubarão e na segunda fase três participantes fizeram parte da intervenção fisioterapêutica. Os dados foram tratados com estatística descritiva (média e desvio-padrão), inferencial (teste de wilcoxon com p < 0,05) e correlação de Pearson. Resultados: Na primeira fase, relacionando a Escala de Epworth com as variáveis analisadas, as mulheres apresentaram maior propensão ao sono, os indivíduos acima de 50 anos e com IMC $>25$ apresentaram maior sonolência excessiva diurna e a qualidade de vida foi menor em indivíduos com maior pontuação na escala. Nos resultados pré e pós-intervenção fisioterapêutica, descritos na segunda fase, dois indivíduos diminuíram a pontuação na Escala de Epworth e um aumentou, houve aumento na pontuação de SF36 relacionado à qualidade de vida, dois indivíduos aumentaram o IMC e um diminuiu, sendo que todos os participantes diminuíram a circunferência do pescoço e a protusão de cabeça. Conclusão: A fisioterapia pode proporcionar bons resultados no tratamento do ronco, contribuindo com a diminuição da sonolência excessiva diurna e melhora da qualidade de vida.

Palavras-chave: Ronco. Sonolência excessiva diurna. Qualidade de vida. Fisioterapia.

\section{Abstract}

Introduction: The snoring is characterized as the sound vibration of the soft tissues of the pharynx, due to difficulty is that the air as it passes through the upper airway with partial obstruction. Objective: To examine the effectiveness of physiotherapeutic intervention in adult apnea, attended clinic school at the Universidade do Sul de Santa Catarina (Unisul). Method: It 
is an explanatory research, quantitative and almost experimental. In the first phase the sample was composed of 7 persons residing in the city of Tubarão and the second phase 3 participants were part of physiotherapeutic intervention. The data were treated with descriptive statistics (mean and standard deviation), inferential (Wilcoxon test with $p<0.05$ ) and Pearson correlation. Results: In the first phase, linking the scale of Epworth with the variables analyzed, women had greater propensity to sleep, individuals over 50 years and with BMI > 25 had higher excessive daytime sleepiness and quality of life was lower in individuals with higher score on the scale. The results before and after physiotherapeutic intervention, described in the second phase, 2 subjects decreased the score on the scale of Epworth and 1 increased, there was an increase in scores of SF36-related quality of life, 2 individuals increased BMI and 1 reduced and all participants decreased the circumference of the neck and head of protrusion. Conclusion: The physiotherapy can provide good results in the treatment of snoring, contributing to the reduction of excessive daytime sleepiness and better life quality.

Keywords: Snore. Excessive daytime sleepiness. Life quality. Physiotherapy.

\section{Introdução}

O ronco passou a ser considerado um problema de saúde, visto que os pacientes roncadores procuram tratamento por diversos fatores, os quais, de acordo com o Consenso em Ronco e Apneia do Sono, vão desde os "[....] ruídos que interferem no sono do(a) companheiro(a), até aqueles sintomas como sonolência diurna excessiva, cansaço, fadiga e condições sistêmicas associadas [....]”, sendo que estes fatores interferem na qualidade de vida do indivíduo $(1,2)$. Segundo a Organização Mundial da Saúde (OMS), qualidade de vida é “[....] a percepção do indivíduo de sua posição na vida [....] quanto à cultura e valores" ${ }^{\prime}$ e o ronco reflete negativamente no relacionamento familiar, social, no rendimento intelectual e profissional $(3,4)$.

A Sociedade Brasileira do Sono define o ronco como a vibração dos tecidos moles da faringe, localizados entre a língua e palato, surgindo durante o sono por causa da dificuldade que o ar encontra ao passar pela via aérea superior, espaço desde o nariz até as cordas vocais com obstrução parcial (5). Strohl (6) afirma que o ronco "[....] é resultante de uma limitação de fluxo e um aumento dos esforços respiratórios e [...] produzem hipoventilação e/ou despertares do sono".

De acordo com alguns estudos $(2,7-10)$, as causas que levam ao ronco podem ser tônus muscular incompetente da musculatura local (palato, língua e faringe), acúmulo de gordura na região do pescoço (obesidade), hipoplasia de maxila ou mandíbula, macroglossia, hipertrofia de amígdalas ou adenoide, efeito de sedativos, tranquilizantes, álcool, comprimento excessivo do palato e/ou úvula, além do posicionamento ao dormir.

Estima-se que $77 \%$ dos pacientes roncadores não sabem que roncam (6). Porém, a incidência é que o ronco está presente em 5 a 50\% da população e tende a aumentar com a idade (11). Em geral, o ronco acomete $45 \%$ dos homens e 35\% das mulheres acima dos 65 anos (6).

A grande maioria dos estudos e técnicas desenvolvidas por profissionais da área da saúde como alternativas para o tratamento do ronco, são baseadas em procedimentos cirúrgicos. O procedimento não invasivo, considerado o tratamento mais efetivo até o momento, é a Pressão Positiva Contínua nas Vias Aéreas, do inglês Continuous Positive Airway Pressure (CPAP). Silva e Pachito (12) afirmam que "[...] o ar sob pressão penetrando nas vias aéreas impede o colapso das paredes musculares faringianas, evitando a ocorrência das apneias, hipopneias e de respiração com esforço aumentado". Porém, gera desconfortos ao indivíduo, dificultando sua adesão.

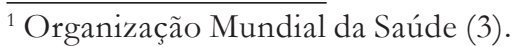


Desta forma, o objetivo geral do trabalho foi analisar a eficácia da intervenção fisioterapêutica em adultos roncadores, atendidos na Clínica-Escola de Fisioterapia da Universidade do Sul de Santa Catarina (Unisul). E, como objetivos específicos, comparar os pontos obtidos na Escala Epworth com gênero, idade, índice de massa corporal (IMC) e qualidade de vida; avaliar a graduação da sonolência diurna pela Escala de Epworth pré e pós-intervenção fisioterapêutica; avaliar a qualidade de vida do portador de ronco pelo Medical Octcomes Study 36 - Item Short-Form Health Survey (SF36) pré e pósintervenção fisioterapêutica; correlacionar o IMC e circunferência do pescoço pré e pós-intervenção fisioterapêutica; analisar a protusão de cabeça pré e pós-intervenção fisioterapêutica.

No presente estudo, os adultos roncadores obtiveram resultados positivos com relação aos objetivos traçados, beneficiando-se com uma abordagem terapêutica não invasiva.

\section{Materiais e métodos}

A pesquisa, quanto ao nível, é classificada como explicativa. Esta estabelece uma relação de causalidade entre as variáveis em estudo. Assim, preocupa-se em identificar os fatores que contribuem ou determinam a ocorrência dos fatos ou a maneira que acontecem (13); quanto à abordagem, classifica-se como quantitativa; quanto ao procedimento utilizado, a pesquisa está classificada como quase experimental. Este tipo de pesquisa constitui-se numa modalidade substancialmente mais fraca, em que a perda da distribuição aleatória não pode garantir que os grupos, experimental e controle, sejam iguais (14). É considerado como o tipo de pesquisa em que não há pleno controle da aplicação dos estilos experimentais que tornem possível um autêntico experimento (15).

A população foi constituída por indivíduos roncadores residentes da cidade de Tubarão e a amostra foi do tipo não probabilística e acidental, sendo selecionada a partir da divulgação da pesquisa por cartazes e do Sistema Integrado de Comunicação (SIC) da Unisul (16). De acordo com o material divulgado, os interessados deveriam estar presentes no Auditório do Bloco da Saúde no dia 24 de março de 2008, para a apresentação do trabalho e convocação dos participantes. Assim, para a primeira fase da pesquisa, a amostra foi composta dentre os demais presentes, por sete indivíduos residentes da cidade de Tubarão, no período entre março e abril de 2008, que preencheram os seguintes critérios de inclusão: adultos com faixa etária de 21 a 65 anos de idade, aceitaram participar da pesquisa e disponibilidade de tempo para participar das palestras de acompanhamento e avaliação.

$\mathrm{Na}$ segunda fase a técnica de amostragem foi do tipo casual simples, onde três dos sete participantes fizeram parte da intervenção fisioterapêutica. Critérios de exclusão: patologia crônica do trato respiratório e indicação cirúrgica para tratamento do ronco.

Todos os indivíduos que compuseram a amostra foram informados sobre todo o procedimento a ser realizado durante a pesquisa e, após esclarecimento, receberam um documento legal, o termo de consentimento livre e esclarecido (TCLE).

Durante a avaliação do paciente, foram utilizados alguns materiais para a coleta de dados, como: ficha de avaliação para a coleta de informações relacionadas ao perfil dos indivíduos que compõem a amostra; máquina fotográfica digital 7.2 mega pixels; corel DRAW ${ }^{\circledR} \mathrm{X} 3$, versão 13.0.0.576 (c) 2005 Corel Corporation, com número de série DR13CER-5809310-CBB; fita métrica de 150 centímetros; balança; estadiômetro; sensy ball ${ }^{\circledR}$; therapeep ${ }^{\circledR}$; $\operatorname{respiron}^{\circledR}$; caneleira de $1 \mathrm{~kg}$. Além dos questionários: Escala de Epworth, sendo aplicada no paciente e utilizada para avaliar a sonolência diurna, quantificando a propensão de o paciente cochilar perante oito situações da vida diária, atribuindo notas de zero a três para cada situação $(17,18)$. Esta, "[...] apresenta um valor máximo de vinte e quatro pontos, sendo considerada sonolência diurna excessiva valores acima de onze pontos"2; e o SF-36, sendo um instrumento de avaliação de qualidade de vida, formado por trinta e seis itens, englobando oito escalas ou componentes: capacidade funcional, aspectos físicos, dor, estado geral da

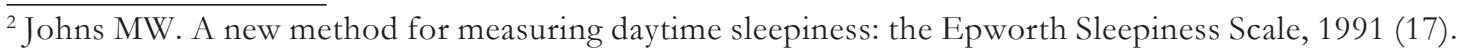


saúde, vitalidade, aspectos sociais, aspectos emocionais e saúde mental (19-23). De acordo com alguns autores (19), existem outros métodos que avaliam de forma mais fidedigna a sonolência, embora para isso haja necessidade de equipamentos específicos e de custo elevado, como a polissonografia.

A partir da aprovação do projeto de pesquisa pelo Comitê de Ética em Pesquisa (CEP) da Unisul, câmpus Regional Sul/unidade Tubarão, protocolo número 08.010.4.08.III, foi realizada a divulgação da pesquisa.

De acordo com o processo de seleção da amostragem, os indivíduos que participaram da reunião e interessaram-se pelo estudo foram convidados a compor a amostra.

$\mathrm{Na}$ avaliação foram coletados dados como pressão arterial, frequência cardíaca e respiratória, peso e altura para calcular o índice de massa corporal (IMC), imagens fotográficas em AP e perfil de tronco superior com o paciente em posição ortostática e em decúbito dorsal, circunferência do pescoço com o paciente sentado e questões relacionadas a hábitos de vida como fumo, álcool, alimentação e posição adotada ao dormir, além da aplicação de questionários referente à sonolência diurna excessiva e qualidade de vida. Nesse período foi entregue e assinado o termo de consentimento livre e esclarecido (TCLE) para todos os participantes.

Após a avaliação, foram analisados quais os indivíduos que apresentavam os critérios de inclusão para a pesquisa e quais destes receberiam intervenção fisioterapêutica, para então se dar início o tratamento proposto pelas pesquisadoras. Os encontros com o grupo tratado foram realizados no laboratório de Fisioterapia em data e horário previamente agendados. E os demais participantes, caracterizado como tratamento incompleto, seguiram apenas as orientações relacionadas ao posicionamento ao dormir, controle de álcool, controle do fumo, peso etc., ou seja, assuntos abordados nas palestras realizadas semanalmente para todos os indivíduos da pesquisa (grupo tratado e tratamento incompleto). Foram realizadas seis palestras durante o decorrer da pesquisa, sempre às 18 horas das segundas-feiras, durante aproximadamente uma hora e trinta minutos, tendo como tema, respectivamente: "ronco X qualidade de vida", ministrada pelas pesquisadoras; "exercício X ronco", ministrada pelo orientador da pesquisa; "nutrição X ronco", ministrada por uma docente do curso de Nutrição; farmácia $\mathrm{X}$ ronco, ministrada por uma docente e duas acadêmicas do curso de Farmácia; "psicologia X ronco", ministrada por dois acadêmicos do curso de Psicologia e "odontologia X ronco", ministrada por uma docente do curso de Odontologia.

Os atendimentos foram realizados em grupos no período entre abril e junho de 2008, sendo que cada atendimento teve duração de 60 minutos, duas vezes por semana, até completar dez atendimentos. $\mathrm{O}$ atendimento foi subdividido em 5 minutos de aquecimento, 10 minutos de alongamento, 40 minutos de treino de resistência muscular e 5 minutos de relaxamento.

No aquecimento, o paciente em posição ortostática realizou flexão lateral de tronco com o membro superior ipsilateral com flexão de cúbito e rotação interna e o membro superior contralateral com extensão de cúbito e abdução; em seguida invertendo o lado, seguindo uma série de dez movimentos. Após, foram realizados movimentos de circundução da cintura escapular bilateral composto de uma série de dez movimentos para a frente e uma série de dez movimentos para trás. Sentados em bolas terapêuticas, os participantes realizaram automassagem, por meio de movimentos circulares com auxílio da sensy ball ${ }^{\circledR}$ nas regiões de trapézio superior, escalenos e esternocleidomastoideo, durante 3 minutos.

Com os pacientes sentados em frente ao espelho, foi realizado alongamento da unidade cabeça-pescoço de forma ativa, mantendo por 20 segundos cada posição. Esses movimentos compreendem flexão cervical, extensão cervical, inclinação lateral cervical para a direita, inclinação lateral cervical para a esquerda, rotação cervical para a direita e rotação cervical para a esquerda.

Foram realizados também exercícios de alongamento de membros superiores, os quais compreenderam flexão de ombro associado à flexão de cúbito e rotação externa com auxílio da mão contralateral; extensão de ombro com extensão de cúbito e dedos; flexão de ombro até $90^{\circ}$ com extensão 
de cúbito, extensão de punho e dedos; e flexão de ombro até $90^{\circ}$ com extensão de cúbito, flexão de punho e dedos mantendo por 20 segundos cada posição.

Para o treino de resistência muscular foi realizado o método de Kabat, pelas diagonais D1 e D2 para membros superiores, de forma ativa e sem resistência nos primeiros cinco atendimentos. E, a partir do sexto atendimento, foram realizados com resistência externa utilizando caneleira de $1 \mathrm{~kg}$. A diagonal D1 parte da posição de extensão de cúbito, abdução e rotação interna de ombro, pósterodepressão de escápula, pronação de antebraço, extensão de punho e dedos. Realizando o movimento que compreende a flexão de punho e dedos, supinação de antebraço, flexão, adução e rotação externa de ombro, antero-elevação de escápula, retornando para a posição inicial. A diagonal D2 parte da posição de extensão de cúbito, adução e rotação interna do ombro, antero-depressão de escápula, pronação de antebraço, flexão de punho e dedos. Realizando os movimentos associados de extensão de punho e dedos, supinação de antebraço, flexão, abdução e rotação externa de ombro, seguido do retorno à posição inicial. A unidade cabeça-pescoço deve acompanhar todos os movimentos da extremidade distal (24). Foram realizadas três repetições para cada diagonal, primeiramente com o membro superior direito, seguido do membro superior esquerdo.

Para o próximo exercício, o indivíduo permaneceu sentado em um banco encostado na parede e o pesquisador auxiliou na retificação cervical, fazendo com que permanecesse nessa postura por 5 minutos.

É importante lembrar que a língua é um músculo e também precisa ser fortalecido. Neste caso, foram realizados os seguintes exercícios pelos participantes: colocação da língua reta para fora, colocação da língua para fora para esquerda e depois para a direita, tocar o nariz com a língua, tocar o queixo com a língua, enrolar a língua, elevar a língua, mover a língua lateralmente na boca, tocar a ponta da língua no palato, logo atrás dos dentes da frente, permanecendo 10 segundos em cada posição e recebendo estímulo verbal para fazer força contra a resistência imposta (24).

Para o treino respiratório, foi utilizado um incentivador inspiratório (repiron ${ }^{\circledR}$ ) e um incentivador expiratório com pressão positiva (therapeep ${ }^{\circledR}$ ), sendo realizadas três séries de oito repetições com intervalo de 20 segundos para cada exercício.

Com os indivíduos em duplas e sentados em bolas terapêuticas, foram realizados movimentos circulares com auxílio da sensy ball ${ }^{\circledR}$ nas regiões de trapézio superior, escalenos e esternocleidomastoideo durante cinco minutos, com o objetivo de massagear e relaxar a musculatura exercitada. As duplas foram formadas para que um participante aplicasse a massagem no outro.

Para o tratamento dos dados na primeira fase da pesquisa, foi realizada estatística descritiva (média e desvio-padrão) e inferencial (teste de Wilcoxon com p $<0,05$ ) para comparar gênero e Escala Epworth. E, para analisar a Escala Epworth com idade, IMC e qualidade de vida (SF-36), foi realizada a correlação de Pearson. Na segunda fase do trabalho, os resultados foram tratados com média e desviopadrão, onde foram demonstrados IMC, circunferência do pescoço, qualidade de vida (SF-36) e Escala Epworth pré e pós-intervenção fisioterapêutica. A apresentação dos dados foi feita através de gráficos de colunas, dispersão e linhas.

\section{Resultados}

Foram avaliados sete indivíduos que participaram da primeira fase da pesquisa, sendo a amostra constituída por quatro homens e três mulheres com idade média de 51,3 \pm 5,6 anos. Na segunda fase, permaneceram três indivíduos que realizaram os dez atendimentos de fisioterapia, sendo a amostra composta por três homens com idade média de $54 \pm 3,5$ anos.

$\mathrm{Na}$ primeira fase, foi realizada a comparação dos pontos obtidos na Escala Epworth com gênero, idade, IMC e qualidade de vida. 


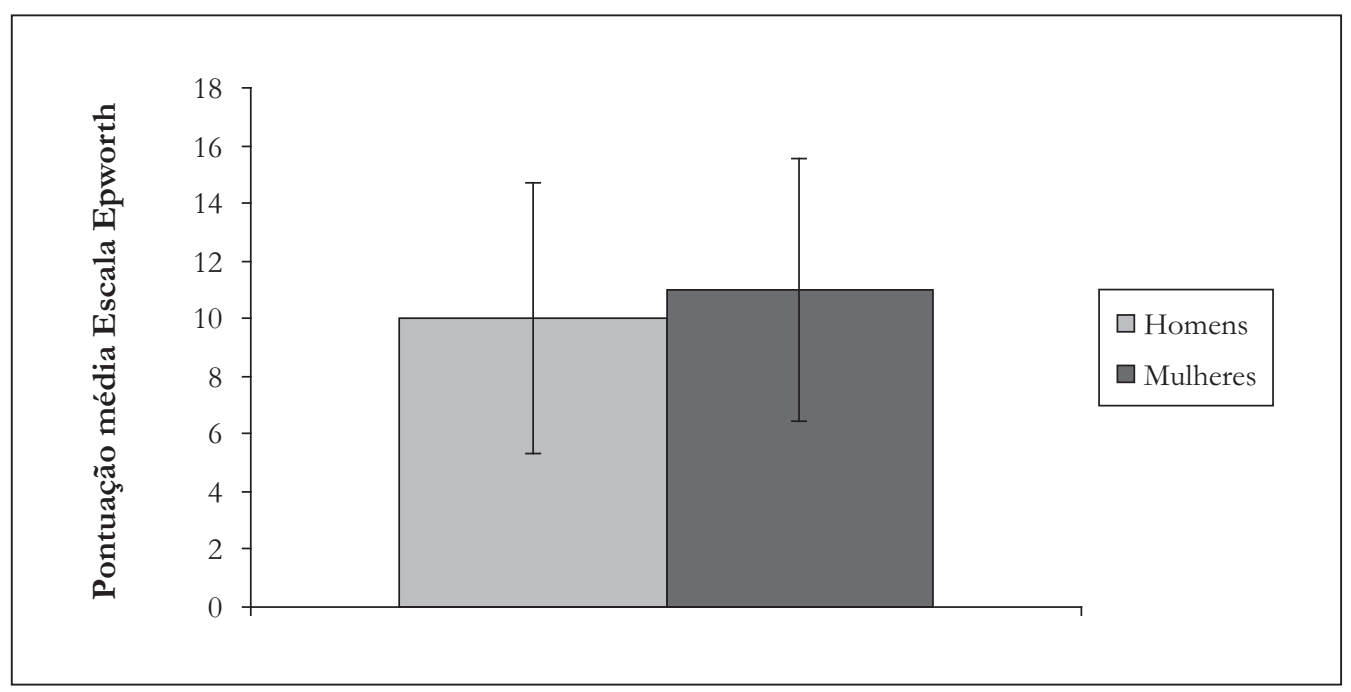

Gráfico 1 - Gênero e pontuação média na Escala de Epworth

De acordo com alguns autores (17-19), a Escala de Epworth é uma forma subjetiva e de baixo custo para a avaliação da sonolência diurna excessiva, sendo este um dos sintomas referidos pelo adulto roncador. Comparando a Escala de Epworth com gênero, é possível observar no Gráfico 1 que as mulheres referiram maior propensão ao sono em algumas situações descritas nesta escala. Supõe-se que esse resultado seja por causa do tamanho da amostra ser pequena e não homogênea. Porém, estudos relacionados ao ronco $(6,25,26)$ apontam o gênero masculino como mais acometido, sendo que a relação homem/mulher, em grande parte das estimativas, varia entre 2:1 e 4:1.

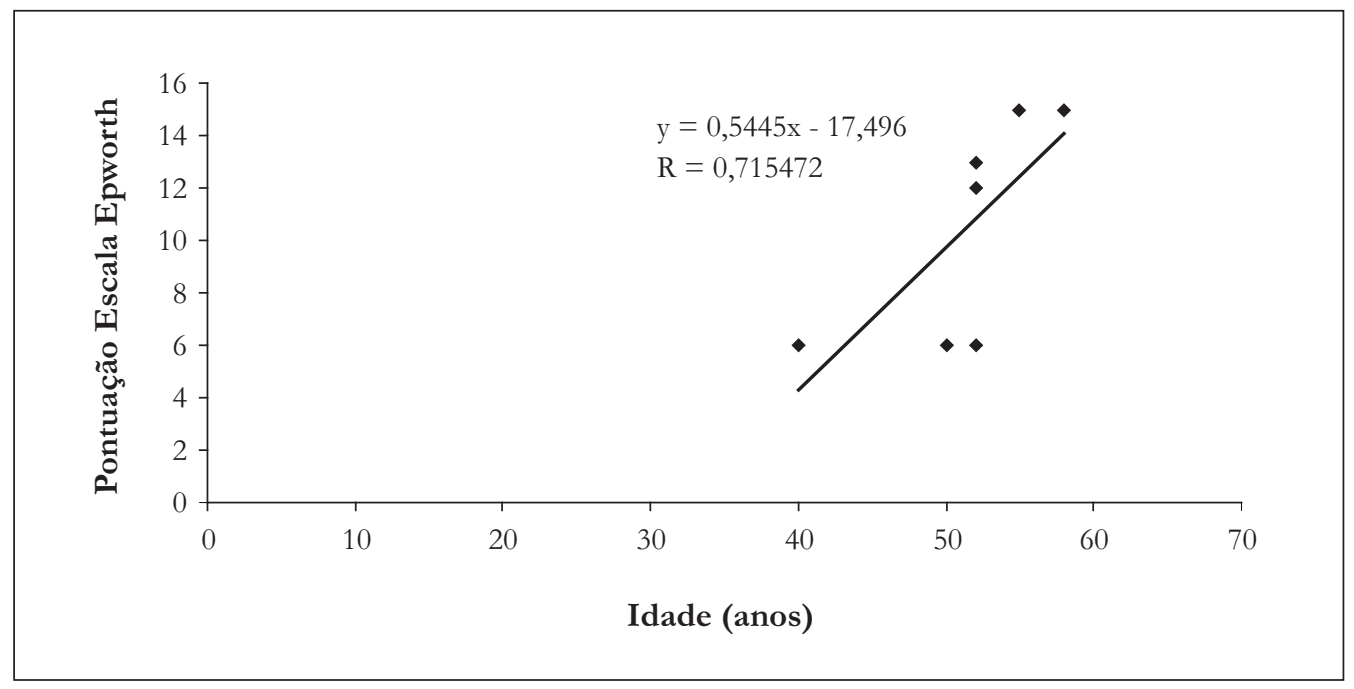

Gráfico 2 - Idade e pontuação na Escala de Epworth

$\mathrm{Na}$ comparação entre a Escala de Epworth e a idade, conforme ilustrado no Gráfico 2, os pontos obtidos foram mais elevados em indivíduos com mais de 50 anos. Logo, neste estudo, pode-se afirmar que a sonolência diurna excessiva, como característica do adulto roncador, está relacionada com o aumento da idade, evidenciada pela correlação positiva e forte, com significância estatística p $<0,05$. De acordo com pesquisas já realizadas, o ronco tende a aumentar com a idade (6, 11). Estudos direcionados à prevalência foram realizados em uma população de homens e mulheres com idade entre 20 e 100 anos, os quais foram separados em subgrupos e apresentaram como resultado a maior prevalência de ronco em mulheres acima de 65 anos e nos homens entre 45 e 64 anos $(26,27)$. 


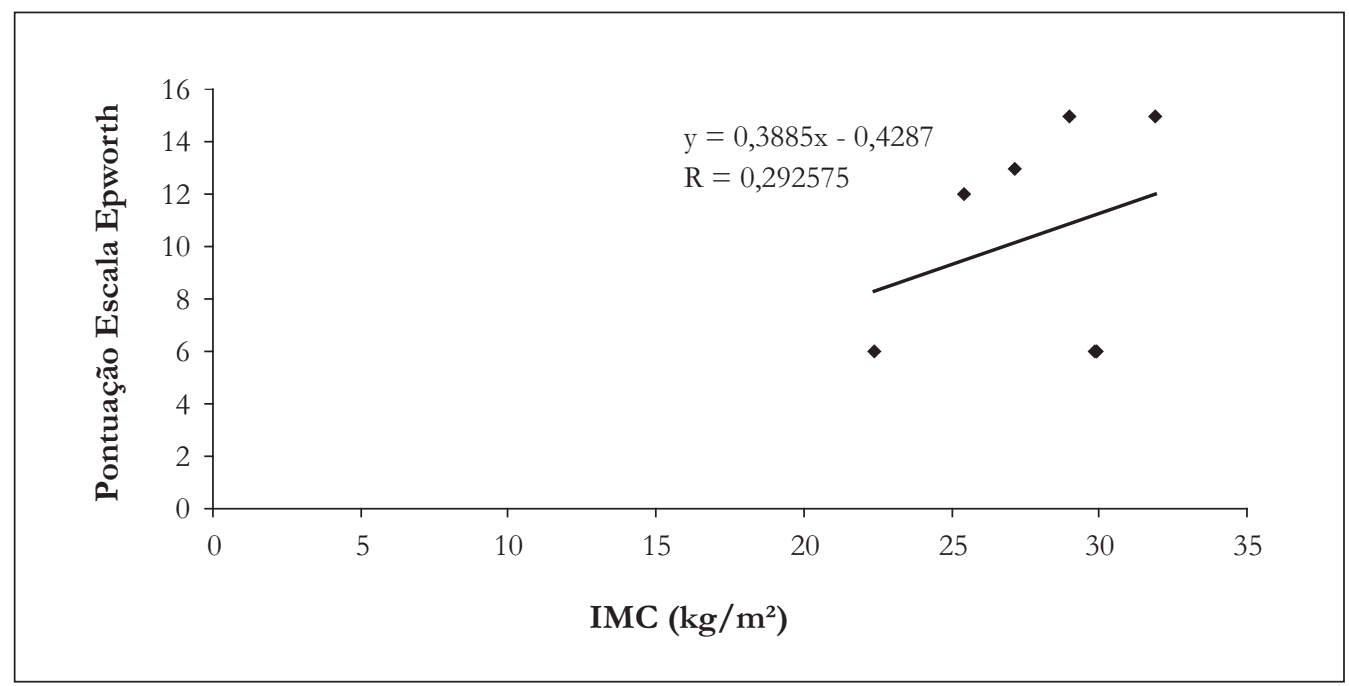

Gráfico 3 - IMC e pontuação na Escala de Epworth

Na comparação entre a Escala de Epworth e o IMC, conforme ilustrado no Gráfico 3 e de acordo com alguns autores $(25,28)$, indivíduos com IMC $>25$, já considerado obesidade, apresentam maior prevalência ao ronco e, consequentemente, maior propensão à sonolência diurna excessiva, evidenciada pela correlação positiva fraca a moderada. A obesidade, portanto, está diretamente relacionada com o ronco, sendo que, quanto maior o IMC, maior acúmulo de gordura na região das vias aéreas superiores, situação tratada como uma das causas de transtornos respiratórios do sono e ronco $(1,4,9,25,28,29)$. Esse fato justifica a importância da análise do IMC nesses indivíduos (20).

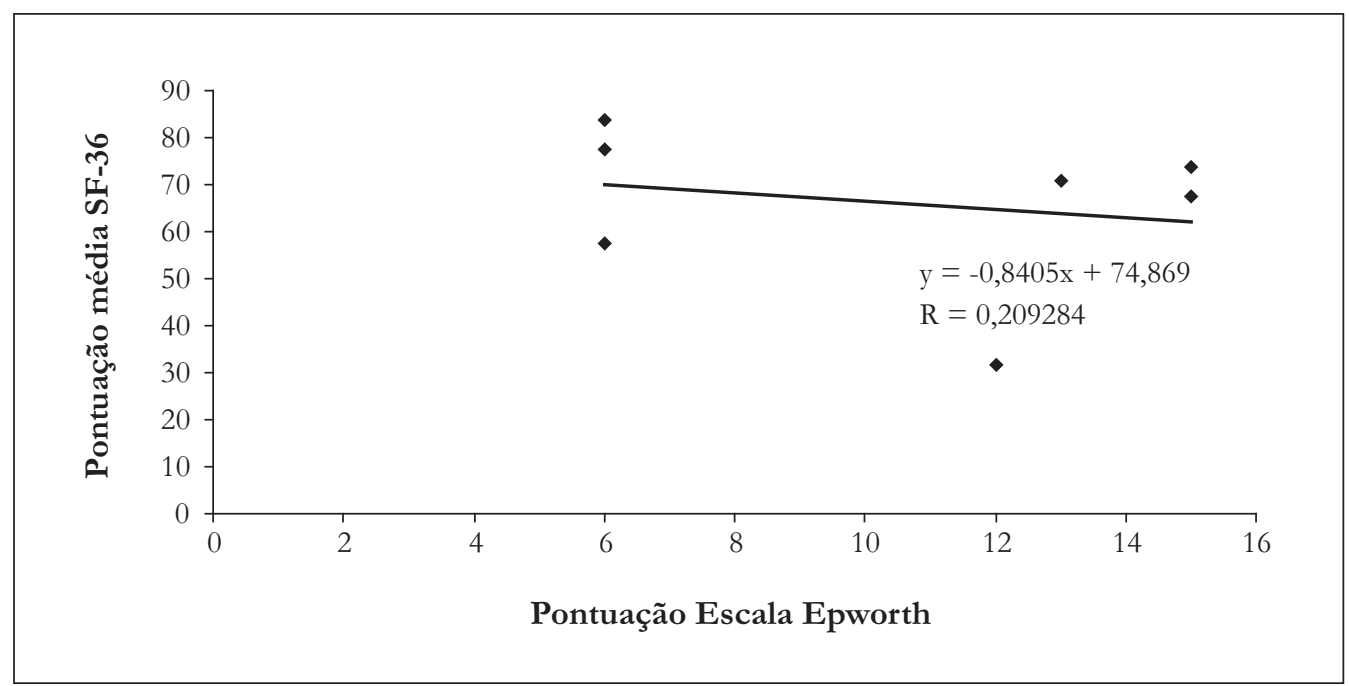

Gráfico 4 - Pontuação na Escala de Epworth e pontuação média no SF-36

Comparando a Escala de Epworth com o SF-36, ilustrado no Gráfico 4, houve correlação negativa fraca à moderada entre a qualidade de vida e a sonolência diurna excessiva. Porém, estudos apontam que há correlação entre essas variáveis, pois o ronco interfere no sono, logo, está associado à sonolência diurna excessiva, perda subjetiva da capacidade de trabalho, cefaleia matinal e maior índice de acidentes de trânsito, predispondo à uma má qualidade de vida (2, 30-32).

Para a segunda fase foram descritos os pontos obtidos na Escala Epworth e qualidade de vida (SF-36), correlação entre o IMC e circunferência do pescoço e análise da protusão de cabeça pré e pós-intervenção fisioterapêutica. 


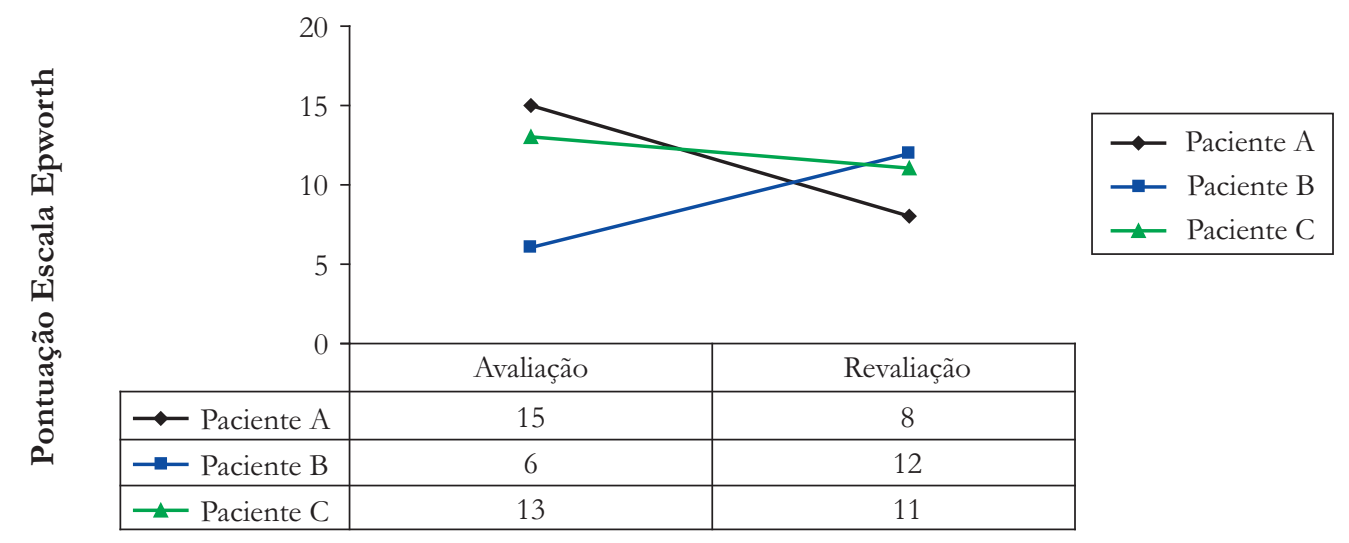

Gráfico 5 - Pontuação na Escala de Epworth pré e pós-intervenção fisioterapêutica

Na descrição dos pontos da Escala de Epworth, conforme Gráfico 5, dos três indivíduos que participaram do estudo, dois diminuíram a pontuação nesta escala e um aumentou após a intervenção fisioterapêutica. Sendo que outros fatores podem estar associados, como, por exemplo, aumento na carga de trabalho, conforme relatado pelo indivíduo que obteve a pontuação elevada após a intervenção.

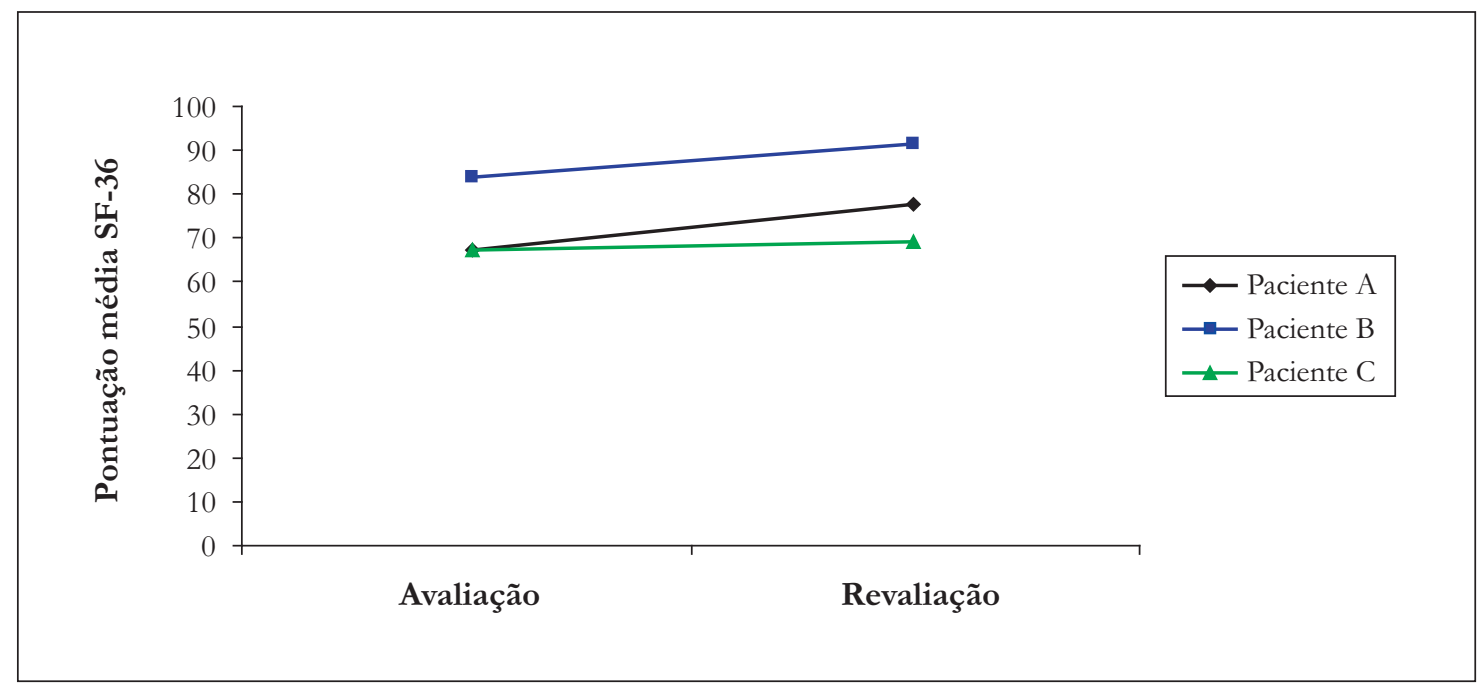

Gráfico 6 - Pontuação média no SF-36 pré e pós-intervenção fisioterapêutica

A qualidade de vida descrita por meio do questionário SF36, como se pode observar no Gráfico 6, atingiu níveis mais elevados após a intervenção fisioterapêutica. 


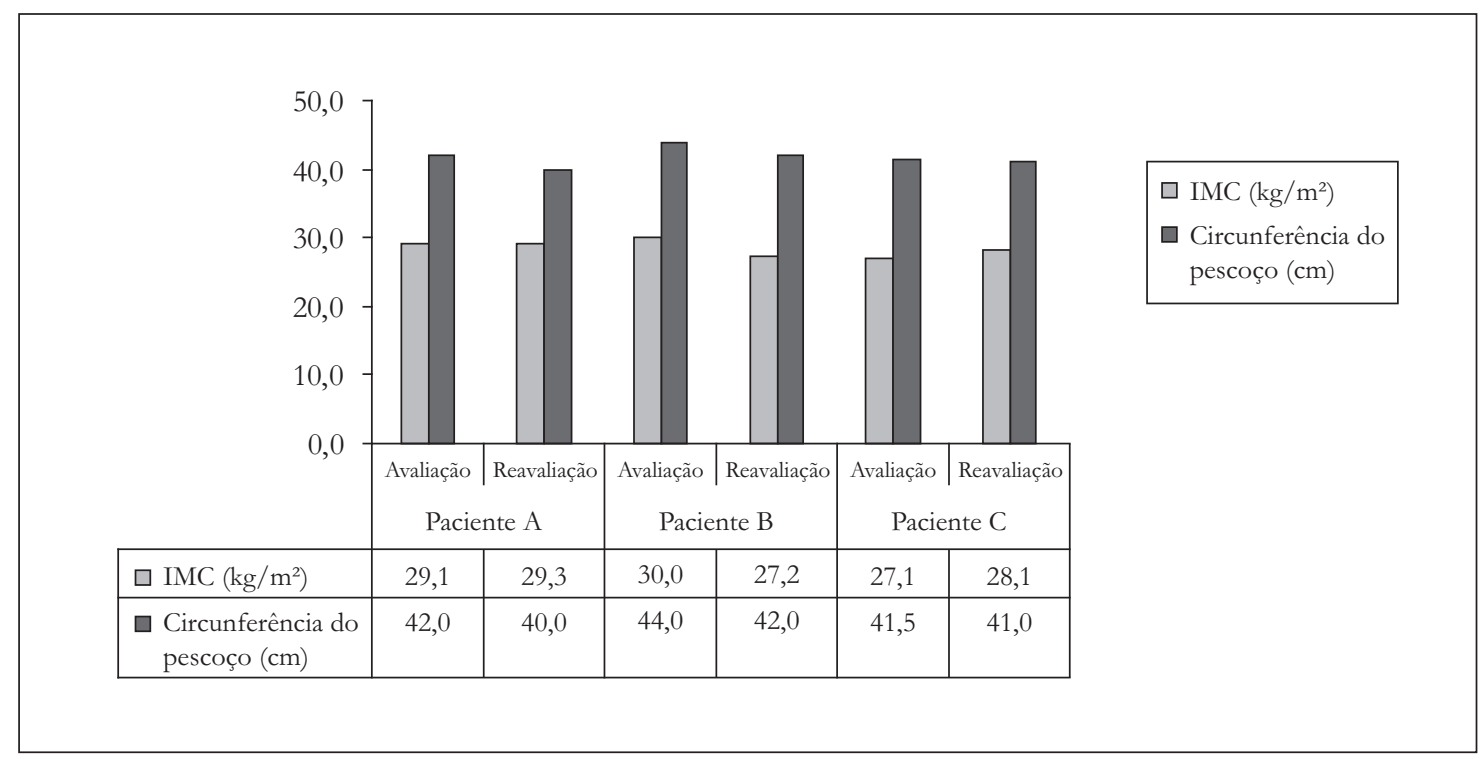

Gráfico 7 - IMC $(\mathrm{kg} / \mathrm{m} 2)$ e circunferência do pescoço $(\mathrm{cm})$ pré e pós-intervenção fisioterapêutica

Conforme Gráfico 7, apesar de um dos participantes da pesquisa ter diminuído o IMC e dois terem aumentado após a intervenção fisioterapêutica, todos obtiveram valores menores com relação à circunferência do pescoço em sua reavaliação. Cabe ressaltar que todos os indivíduos que fizeram parte da amostra participaram das palestras multidisciplinares de acompanhamento, incluindo o curso de Nutrição, e estavam cientes da importância da perda de peso associado a qualquer tratamento adotado para o ronco. Estudos apontam que a deposição aumentada de gordura na região do pescoço ou adjacente à via aérea superior, seja responsável pela obstrução do fluxo aéreo total (apneia) em obesos $(33,34)$. Borges e Paschoal $(35)$ afirmam que a perda de peso é fundamental em indivíduos com IMC elevado, podendo até curar a síndrome da apneia/hipopneia do sono.

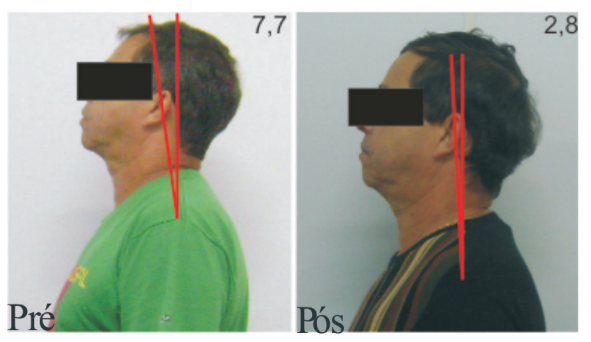

Figura 1 - Paciente A

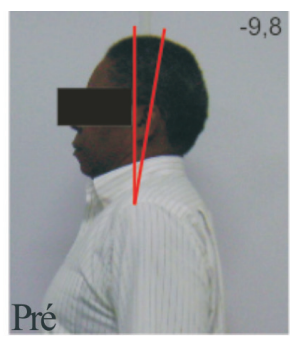

Figura 2 - Paciente B

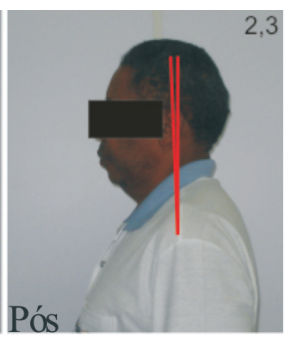

Pré

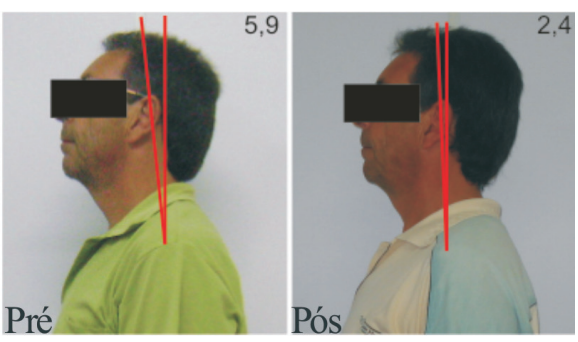

Figura 3 - Paciente C

É possível observar nas Figuras 1, 2 e 3 que houve diminuição na protusão de cabeça pósintervenção fisioterapêutica. Os indivíduos roncadores, em sua maioria, apresentam protusão de cabeça, devendo, então, ser este um dos itens a compor a avaliação. De acordo com Makofsky (36), as alterações presentes no adulto roncador compreendem a resposta estabilizadora anterior da cabeça, no qual a "[...] laringe é tracionada para cima e para frente, as escapulas sofrem protração, a parede torácica anterior sofre depressão e os ombros e as costelas perdem mobilidade". 
Durante esse estudo foram realizadas palestras semanalmente para todos os indivíduos da pesquisa, envolvendo diversos profissionais da área da saúde, com objetivo de repassar orientações relacionadas a fatores que predispõem ao ronco, como posicionamento ao dormir, álcool, fumo, medicamentos, peso, entre outros.

Estudo de base populacional foi realizado com 3.136 adultos, sendo encontrada prevalência de 50,5\% de ronco, acometendo mais os homens, idosos, tabagistas, alcoolistas e obesos (28). Nesse estudo, os autores destacam que fatores de risco como sexo e idade, não são modificados, porém o tabagismo, alcoolismo e obesidade devem ser identificados e tratados (28).

De acordo com Borges e Paschoal (35), para o sucesso do tratamento o indivíduo necessita de mudanças drásticas no modo de vida e isso inclui todos os fatores de risco modificáveis, como evitar o decúbito dorsal, abster-se de fumo e álcool, controle de medicamentos e peso.

Além disso, existe um conjunto de medidas denominadas higiene do sono, as quais são importantes para se obter um sono tranquilo e restaurador. Compreende dormir em ambiente escuro e silencioso, evitar o calor e o frio excessivo no quarto, não dormir com fome, mas evitar refeições pesadas e praticar exercícios regularmente (37). Segundo a American Sleep Disorders Association, o exercício físico regular é considerado uma intervenção não farmacológica utilizada como objetivo de melhorar a qualidade do sono ${ }^{3}$. Essas medidas foram abordadas durante as palestras, de forma multidisciplinar, e os participantes procuraram aderir a esses hábitos, a fim de auxiliar no tratamento do ronco.

Em nosso estudo, após os 10 atendimentos fisioterapêuticos, os participantes obtiveram melhora da qualidade de vida e sonolência excessiva diurna, além da diminuição da circunferência do pescoço e da protusão de cabeça. Os participantes referiram melhora em vários aspectos, como a diminuição do cansaço e a respiração mais leve. Além disso, os familiares perceberam a diminuição na intensidade do ronco.

\section{Considerações finais}

O ronco é um assunto que tem sido discutido com frequência na atualidade. Por se tratar de um assunto relativamente novo, diversos profissionais estão se envolvendo em busca de novas abordagens terapêuticas. Logo, para que o adulto roncador obtenha bom resultado em seu tratamento, é importante a utilização de uma abordagem multidisciplinar envolvendo diversas áreas da saúde, como a Medicina, Fisioterapia, Odontologia, Fonoaudiologia, Nutrição e Psicologia, as quais estão se dedicando a cada dia na busca da melhor terapêutica para o portador desta patologia.

Em nosso estudo pôde-se constatar que a intervenção fisioterapêutica pode proporcionar bons resultados no tratamento de adultos roncadores, beneficiando o indivíduo com uma abordagem terapêutica não invasiva, contribuindo, assim, com a diminuição da sonolência diurna excessiva e uma melhora da qualidade de vida.

Os participantes desta pesquisa obtiveram, ainda, outros resultados positivos, como a diminuição da circunferência do pescoço e da protusão de cabeça, modificando seu perfil postural e, consequentemente, melhorando a aeração de vias aéreas superiores.

Sugere-se a realização de novos estudos na área de Fisioterapia, relacionados ao tratamento

de adultos roncadores, utilizando uma amostra e um tempo de intervenção maiores para resultados mais significativos.

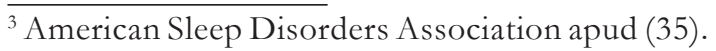




\section{Referências}

1. Urschitz MS, Guenther A, Eitner S, Urschitz-Duprat PM, Schlaud M, Ipsiroglu OS, et al. Risk factors and natural history of habitual snoring. Chest Journal. 2004 [cited 2008 Set 28];126:790-800. Available from: http://www.chestjournal.org/cgi/content/

2. Sociedade Brasileira de Sono, Sociedade Brasileira de Rinologia, Sociedade Brasileira de Otorrinolaringologia. I Consenso em ronco e apnéia do sono [Internet]. São Paulo: Sociedade Brasileira de Sono. 2000 [acesso 28 set 2007]. Disponível em: http://www.sbsono. com.br/hypnos/IConsensoemRoncoeApneiadoSono.pdf

3. Fleck MPA. O instrumento de avaliação de qualidade de vida da Organização Mundial da Saúde (WHOQOL100): características e perspectivas. Ciênc Saúde Coletiva. 2000 [acesso 28 set. 2007];5(1):34-9. Disponível em: http:/ / www.scielosp.org/scielo.php?script =sci_arttext\&pid=S1413-81232000000100004\&lng=\&nrm=isso

4. Ito FA, Ito RT, Moraes NM, Sakima T, Bezerra MLS, Meirelles RC. Condutas terapêuticas para tratamento da síndrome da apnéia e hipopnéia obstrutiva do sono (SAHOS) e da síndrome da resistência das vias aéreas superiores (SRVAS) com enfoque no aparelho anti-ronco (AAR-ITO). Rev Dent Press Ortodon Ortop Facial. 2005 [acesso 16 nov. 2007];10(4):28. Disponível em: http://www.scielo.br/ scielo.php?script $=$ sci_arttext\&pid $=\mathrm{S} 141554192005000400015 \& \operatorname{lng}=\mathrm{pt} \& \mathrm{nrm}=$ iso

5. Sociedade Brasileira do Sono. Ronco. [acesso 16 nov. 2007]. São Paulo: Sociedade Brasileira do Sono. Disponível em: http:/ /www.sbsono.com.br/noticias/ronco.php

6. Strohl KP. Síndrome de hipopnéia-apnéia do sono obstrutiva. In: Goldman L, Ausiello DA, Cecil R. Cecil tratado de medicina interna. Rio de Janeiro: Elsevier; 2005. p. 664-8.

7. Balbani APS, Formigoni GGS. Ronco e síndrome da apnéia obstrutiva do sono. Rev Assoc Méd Brás. 1999 [acesso 2 nov. 2007];45(3):273-8. Disponível em: http://www.scielo.br/pdf/ramb/v45n3/1660.pdf

8. Goldman L, Ausielo DA, Cecil R. Síndrome de hipopnéia-apnéia do sono obstrutiva e doença das vias respiratórias altas. In: Goldman L, Ausielo DA, Cecil R. Cecil tratado de medicina interna. 22a ed. Rio de Janeiro: Elsevier; 2005. p. 2845-6.

9. Braver HM, Block AJ, Perri MG. Treatment for snoring: combined weigth loss, sleeping on side, and nasal spray. Chest Journal. 1995 [cited 2007 Out 26];107:1283-8. Available from: http:/ /www.chestjournal.org/ cgi/content/abstract/107/5/1283

10. Skinner MA, Kingshot RN, Jones DR, Taylor DR. Lack of efficacy for a cervicomandibular support collar in the management of obstructive sleep apnea. Chest Journal. 2004 [cited 2007 Out 26];125:118. Available from: http://www.chestjournal. org/cgi/content/abstract/125/1/118

11. Dibbern RS, Thuler ER, Fomin D, Oliveira JAA. Resultados preliminares do tratamento dos pacientes submetidos a radiofreqüência (baixa freqüência, somnoplastia) no ronco e apnéia leve do sono. Rev Bras Otorrinolaringol. 2002 [acesso 29 set. 2007];68(1):752-6. Disponível em: http://www.scielo.br/ scielo.php?script=sci_arttext\&pid=S0 034-72992002000100017\&lng=PT

12. Silva GA, Pachito DV. Abordagem terapêutica dos distúrbios respiratórios do sono tratamento com ventilação não invasiva (CPAP, BIPAP E AUTO-CPAP). Medicina (Ribeirão Preto). 2006 [acesso 29 set. 2007];39(2):212-7. Disponível em: http://www.fmrp.usp.br/ revista/2006/vol39n2/6_cpap_bipap_e_autocpap.pdf

13. Santos AR. Metodologia científica: a construção do conhecimento. 3a ed. Rio de Janeiro: DP\&A; 2000.

14. Gil AC. Como elaborar projetos de pesquisa. 4a ed. São Paulo: Atlas; 2002.

15. Campbell DT. Delineamentos experimentais e quase experimentais de pesquisa. São Paulo: Ed. da EDUSP; 1979. 
16. Sistema Integrado de Comunicação. Pesquisa propõe novo tratamento para roncadores. UNISUL hoje. 3 jun. 2008 às 02:19 [acesso 7 set. 2008]. Disponível em: http:/ /www.unisul.br/content/jornalunisulhoje/ home/integradanoticia.cfm?objectid=C30513EC-3048-6857-88B7E69AA807CE6D\&secao=Pesquisa

17. Ferreira GDP, Ramos FA, Fomin DS, Alóe F, Pinto JA. Ronco e apnéia-hipopnéia obstrutiva do sono: indicações da somnoplastia e da uvulopalatofaringoplastia com laser de CO2. Arquivos internacionais de otorrinolaringologia. 2001 [acesso 1 out. 2007];5(4):4 . Disponível em: http:/ /www.arquivosdeorl.org.br/ conteudo/acervo_port.asp?id=173

18. Boari L, Cavalcanti CM, Bannwart SRFD, Sofia OB, Dolci JEL. Avaliação da Escala de Epworth em pacientes com a síndrome da apnéia e hipopnéia obstrutiva do sono. Rev Bras Otorrinolaringol. 2004 [acesso 1 out. 2007];70(6):752-6. Disponível em: http://www.scielo.br/scielo.php?script=sci_arttext\&pid=S003472992004000600007

19. Sobrinho, DGR, Roizenblatt S, Lopes AC, Teixeira RCA, Tufik S. Estudo da capacidade de manter o alerta em pacientes com fibromialgia por meio do teste da manutenção da vigília Rev Bras Reumatol. 2008 [acesso 8 out. 2008];48(1). Disponível em: http://www.scielo.br/scielo.php?script=sci_arttext\&pid=S0482$50042008000100004 \& \operatorname{lng}=\& n r m=$ iso\&tlng=

20. 20 Gondim LMA, Matumoto LM; Melo Júnior MAC, Bittencourt S, Ribeiro UJ. Estudo comparativo da história clínica e da polissonografia na síndrome da apnéia/ hipopnéia obstrutiva do sono. Rev Bras Otorrinolaringol. 2007 [acesso 22 set. 2008];73(6):8. Disponível em: http://www.scielo.br/scielo.php? script $=$ sci_arttext\&pid $=$ S0034-72992007000600003\&lng=pt\&nrm=iso

21. Ciconelli RM, Ferraz MB, Santos W, Meinão I, Quaresma MR. Tradução para o português e validação do questionário genérico de avaliação da qualidade de vida SF-36 (Brasil SF- 36). Rev Bras Reumatol. 1999;39(3):5.

22. Ciconelli RM. Tradução para o português e validação do questionário genérico de avaliação da qualidade de vida "Medical Outcomes Study 36- Item Short- Form Health Survey (SF-36)" [Tese na Internet]. São Paulo: Universidade Federal de São Paulo; 1997. [acesso 16 nov. 2007]. Disponível em: http://64.233.169.104/ search?q=cache:ZsE0F4qH-fsJ:www.ciape.org.br/matdidatico/ciomara/sf36.rtf+\%22Tradu $\%$ C3\%

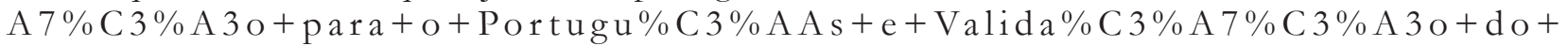
Question $\%$ C $3 \%$ A 1 rio + Ge n $\%$ C $3 \%$ A 9 rico + d e + A va 1 i a $\%$ C $3 \%$ $\mathrm{A} 7 \% \mathrm{C} 3 \% \mathrm{~A} 3 \mathrm{o}+\mathrm{da}+$ Qualidade $+\mathrm{de}+\mathrm{Vida} \% 22 \& \mathrm{hl}=\mathrm{ptBR} \& \mathrm{ct}=\mathrm{clnk} \& \mathrm{~cd}=2 \& \mathrm{gl}=\mathrm{br}$.

23. Yu Li H, Chen NH, Shu YH, Wang PC. Changes in quality of life and respiratory disturbance after extended uvulopalatal flap surgery in patients with obstructive sleep apnea. Arch otolaryngol head neck surg. 2004 [cited 2008 Set 27]. Available from: http://archotol.ama-assn.org/cgi/reprint/130/2/195

24. Smith LK, Weiss EL, Lehmkuhl LD. Cinesiologia clínica de Brunnstrom. 5a ed. São Paulo: Manole; 1997.

25. Martins AB, Tufik S, Moura SMGPT. Síndrome da apnéia-hipopnéia obstrutiva do sono: fisiopatologia. J Bras Pneumol. 2007 [acesso 11 out. 2008];33(1):36-43. Disponível em: http://www.sono.org.br/pdf/ 2007\%20Martins\%20J\%20Bras\%20Pneumol.pdf

26. Bixler EO, Vgontzas AN, Lin H, Have TT, Rein J, Vela-Bueno A, et al. Prevalence of sleep-disordered breathing in women: effects of gender. Am J Respir Crit Care Méd. 2001 [cited 2008 Out 12];163(3):60813. Available from: http://ajrccm. atsjournals.org/cgi/content/full/163/3/608

27. Bixler EO, Vgontzas AN, Lin H, Have TT, Rein J, Tyson K, Kales A. Effects of age on sleep apnea in men. Am J Respir Crit Care Méd. 1998 [cited 2008 Out 12];157(1):144-8. Available from: http:// ajrccm.atsjournals.org/cgi/content/full/157/1/144 
28. Noal RB, Meneses AMB, Canani SF, Siqueira FV. Ronco habitual e apnéia obstrutiva observada em adultos: estudo de base populacional, Pelotas, RS. Rev Saúde Pública. 2008 [acesso 23 set. 2008];42(2). Disponível em: http://www.scielo.br/pdf/ rsp/v42n2/6229.pdf

29. Silva LMP, Aureliano FTS, Motta AR. Atuação fonoaudiológica na síndrome da apnéia e hipopnéia obstrutiva do sono: relato de caso. Rev CEFAC. 2007 [acesso 24 set. 2008];9(4):490-6 . Disponível em: http:// w w w.s c i e lo.br/s c i e lo.ph p ? s c r i pt=s ci_arttext \& p id = S 1516 18462007000400009\&lng=pt\&nrm=iso.doi:10.1590/S1516-1846200700040000 9

30. Küpper DS, Leite MGJ, Nogueira RL, Valera FCP, Oliveira JAA. Tratamento cirúrgico da SAHOS. Medicina, (Ribeirão Preto). 2006 [acesso 29 set. 2007];39(2):218-26. Disponível em: http:/ /www.fmrp.usp.br/revista/ 2006/vol39n2/7_tratamento_cirurgico_sahos.pdf

31. McNicholas WT, Levy P. Sleep-related breathing disorders: definitions and measurements. Eur Respir J. 2000 [cited 2008 Set 23];15:988-989. Available from: http://erj.ersjournals.com/content/vol15/issue6/

32. Bittencourt LRA, Silva RS, Santos RF, Pires MLN, Mello MT. Sonolência excessiva. Rev Bras Psiquiatr. 2005 [acesso 28 set. 2007];27(1):16-21. Disponível em: http:/ /www.scielo.br/pdf/rbp/v27s1/24471.pdf

33. SchäferH,PauleitD,Sudhop T, Gouni-BertholdI, EwigS, Berthold HK. Body fat distribution, serum leptin, and cardiovascular risk factors in men with obstructive sleep apnea. Chest Journal. 2002 [cited 2008 Out 12];122:829-39. Available from: http:/ /www.chestjournal.org/cgi/content/full/122/3/829?maxtoshow=\&HITS=10\&hits=10\&RESULTFORMAT= $\&$ searchid $=1 \&$ FIRSTINDEX $=0 \&$ sortspec $=$ relevance\&volume $=122 \&$ firstpage $=$ 829\&resourcetype $=$ HWCIT

34. Ip MSM, Lam KSL, Ho C, Tsang KWT, Lam W. Serum leptin and vascular risk factors in obstructive sleep apnea. Chest Journal. 2000 [ceted 2008 Out 12];118:580-6. Available from:http:/ /www.chestjournal.org/ cgi $/$ content $/$ full $/ 118 / 3 / 580$ ? maxtoshow $=\& H I T S=10 \&$ hits $=10 \&$ RESULTFORMAT $=\&$ searchid $=1 \&$ FIRSTINDEX $=0 \&$ sortspec $=$ relevance $\&$ volume $=118 \&$ irstpage $=580 \&$ resourcetype $=$ HWCIT

35. Borges PTM, Paschoal JR. Indicação inicial de tratamento em 60 pacientes com distúrbios ventilatórios obstrutivos do sono. Rev Bras Otorrinolaringol. 2005 [acesso 23 set. 2008];71(6). Disponível em: http:// www.scielo.br/scielo.php?pid=S0034-72992005 000600009\&script=sci_arttext

36. Makofsky HW. Coluna vertebral: terapia manual. Rio de Janeiro: Koogan; 2006.

37. Mello MT, Tufik S. Sono e exercício físico. In: Mello MT, Tufik S. Atividade física, exercício físico e aspectos psicobiológicos. Rio de Janeiro: Guanabara Koogan; 2004.

Recebido: 26/01/2009

Received: 01/26/2009

Aprovado: 02/10/2009

Approved: 10/02/2009 